\title{
Cinéma et sciences du psychisme en 1900: la névrose, la paramnésie, la transe
}

Mireille Berton

\section{Summary}

In this article, conceived of in an epistemological perspective, film history and history of psychology intersect in order to show how subject models are circulating around 1900 which are impregnated with scientific culture and social modernity. These models have the special quality of being defined first in pathological terms because they result from a diagnosis of the evils caused by the new industrial and capitalist society. However, the position required by the cinematic apparatus produces a spectator who is described as a subject-machine with the particular psychophysiological states that precisely concern the neurotic, the paramnesiac and the sleepwalker. If our point is to show how close the (para)medical and cinematic subjects are, we will also try to see how far cinema interacts with phenomena related to urbanity and other developments of Western civilisation, the latter being considered as a transforming agent of the psychic apparatus.

Keywords: psychic sciences; neurology; film around 1900

\section{Résumé}

Pensé dans une perspective épistémologique, cet article croise l'histoire du cinéma et l'histoire des sciences du psychisme afin de mettre en évidence la circulation de modèles de subjectivité imprégnés par la culture scientifique et la modernité sociale autour de 1900. Ces modèles présentent la particularité d'être définis d'abord en termes pathologiques car codifiés en fonction d'un diagnostic des méfaits de la nouvelle société industrielle et capitaliste. 
Or, la posture requise par le dispositif cinématographique induit un spectateur qui est décrit comme un sujet-machine en proie à des états psychophysiologiques particuliers qui concernent, précisément, autant le névrosé, le paramnésique que le somnambule. S'il s'agira de démontrer quelles sont les affinités entre le sujet (para)médical et le sujet cinématographique, on tentera également de réfléchir aux interactions du cinéma avec des phénomènes relatifs à l'urbanité et au développement de la civilisation occidentale considérée comme un agent de transformation de l'appareil psychique.

L'histoire du cinéma et l'histoire des sciences du psychisme se rencontrent autour de 1900 à plusieurs niveaux et selon diverses modalités. Un des croisements principaux se noue autour des tentatives de penser la subjectivité relativement à des données conjoncturelles participant à l'émergence de troubles spécifiques du corps et de l'esprit. Menée au sein d'une recherche plus large dessinant les contours d'un champ à construire sur la base d'un dialogue entre histoire du cinéma des premiers temps, théories du dispositif et du spectateur, et histoire des sciences, cette réflexion s'appuie méthodologiquement sur les acquis de l'historiographie du cinéma actuelle ${ }^{1}$. Visant l'élaboration d'une épistémologie du cinéma qui s'inscrit dans la tradition d'une archéologie foucaldienne des savoirs, ces analyses déchiffrent la grille symbolique à travers laquelle le tournant du siècle appréhende divers savoirs, discours, représentations et pratiques, dans le but de rendre compte du rôle joué par le cinéma à l'intérieur des mutations épistémiques engendrées par la modernité . Pour ma part, je m'interroge sur les logiques d'interactions entre les dispositifs d'images animées et les discours médicaux, psychologiques et parapsychologiques, dans un contexte où la modernité sociale, technologique et industrielle façonne un nouvel environnement, ainsi que des habitudes perceptives et des maladies inédites. Il s'agit d'examiner dans quelle mesure et dans quelles circonstances le cinéma - conçu autant comme machine de fabrication que de réception d'images animées - interagit avec un certain nombre de phénomènes relatifs à l'urbanité et au développement de la civilisation occidentale considérée comme un agent de transformation de l'appareil psychique.

1 Cet article est tiré d'une thèse de doctorat en cours intitulée «Le dispositif cinématographique comme modèle épistémologique dans les sciences du psychisme au tournant du $\mathrm{XX}^{\mathrm{e}}$ siècle. L'invention du sujet moderne». Elle est dirigée par le Prof. François Albera à l'Université de Lausanne, au sein de la Section d'Histoire et esthétique du cinéma.

2 Albera/Tortajada 2004; Tortajada 2004. 
Au tournant du $\mathrm{XX}^{\mathrm{e}}$ siècle, les modèles de subjectivité présentent la particularité d'être définis d'abord en termes pathologiques, comme le démontrent les textes qui les codifient en fonction d'un diagnostic des méfaits de la nouvelle société industrielle et capitaliste provoquant asthénie, irritabilité ou suggestibilité. Si ces dispositions touchent en priorité l'habitant des grandes agglomérations et l'usager des technologies du transport, de la communication ou des loisirs (telles que le train, la téléphonie/télégraphie ou les parcs d'attraction), elles regardent également le modèle de subjectivité requis par le dispositif cinématographique, le spectateur étant décrit comme éprouvant, lui aussi, épuisement nerveux, légère transe, surstimulation perceptive ou exaltation visionnaire. Les modes de projection et de réception du film, consistant en une discontinuité séquentielle partiellement gommée par sa mise en mouvement et un bombardement de stimuli ressentis sous la forme d'un choc, seraient donc à la fois le produit et le symbole de cette modernité urbaine. Ma démonstration s'appuiera sur trois exemples d'articulation possible entre les sciences du psychisme et le cinéma, et plus précisément entre les modèles de subjectivité figurant dans la littérature médicale et paramédicale, et les discours qui rendent compte de l'expérience du spectateur du cinéma. Ainsi, on mesurera les affinités existant entre le sujet spectatoriel, et, tour à tour, le sujet névrotique (renvoyant aux pathologies de l'excitabilité sensorielle), le sujet paramnésique (incarnant les aberrations de la mémoire), et le sujet somnambulique (indisposé par des pratiques médiumniques).

\section{Le sujet névrotique}

S'il s'avère possible de mettre en parallèle le sujet névrotique - dans ses déclinaisons hystérique, épileptique ou neurasthénique - avec le sujet spectatoriel, c'est parce que tous deux sont pensés en termes dynamogéniques, conformément à une culture scientifique imprégnée par la seconde révolution industrielle 3 . Depuis les années 1870, la neurasthénie, mal symptomatique de la civilisation occidentale, exprime un dérèglement du système nerveux qui oscillerait entre une tendance «hyponévrique» et «hypernévrique» ${ }^{4}$, tel que mentionné dans le Dictionnaire encyclopédique des sciences médicales. Ainsi, «ce serait [...] à l'excitabilité ou au défaut de rapport entre l'ex-

\footnotetext{
3 J'ai traité ailleurs des liens entre le cinéma et l'imaginaire du corps-machine dans un article à paraître: «L'appareil psychique comme machine électrophysiologique: le cinéma et la psychanalyse à l'aune de la modernité». Actes du Colloque international «Cinema e Psicoanalisi» (Università di Studi di Rome Tre, 26-27 octobre 2006).

4 Brochin 1878, 360.
} 
citabilité et l'excitation, et aux diverses conditions de cette déséquilibration [...] que pourraient être ramenées les diverses formes de la névropathie» 5 . Les nombreuses définitions qui ont été données de la neurasthénie recouvrent une conception du psychisme comme partagé entre asthénie et surtension, d'où la récurrence de syntagmes oxymoriques tels que «passions dépressives $»^{6}$ ou «faiblesse irritable» ${ }^{7}$. Selon le docteur Léon Bouveret, si la neurasthénie consiste en «un affaiblissement durable de la force nerveuse», il faut noter qu' «aux symptômes de dépression [...] sont souvent associés des symptômes d'excitation», donnant ainsi à cette maladie un «double caractère de faiblesse et d'irritabilité» ${ }^{8}$. Dans la même lignée, le médecin lausannois Virgile Borel suggère que la maladie se traduit par «l'exagération, la diminution ou la perversion du mode de réception, de réaction ou d'action initiale du système nerveux sur les agents destinés à l'impressionner (agents physiques, chimiques, vitaux et spirituels)» ${ }^{9}$. Ainsi, une réponse excessive ou insuffisante aux stimuli exogènes ou endogènes peut avoir les mêmes conséquences et entraîner la même maladie, attendu que «le manque d'excitant ou de réaction pourra, par un chemin opposé, arriver aux mêmes résultats» ${ }^{10}$.

Parmi les causes invoquées pour expliquer cette déstabilisation du flux nerveux et énergétique, les médecins citent pêle-mêle la sédentarité, l'éducation «molle et efféminée», les plaisirs de la vie en société, et «l'activité dévorante que suscitent souvent l'ambition et l'action énervante de toutes les passions violentes» ${ }^{11}$. Mettant à rude épreuve les organismes, la neurasthénie concerne tous les «pays civilisés où la lutte pour l'existence, de plus en plus ardente, entretient une activité exagérée de toutes les fonctions du système nerveux ${ }^{12}$. Bouveret met ainsi en exergue l'incidence morbigène de «la vie mondaine dans les grandes villes [qui] constitue un véritable surmenage des sens et du système nerveux», car «elle se compose de plaisirs qui $[\ldots]$ excitent et fatiguent le cerveau sans le reposer jamais» ${ }^{13}$. Le monde moderne, avec son organisation économique faite d'une intense circulation d'objets, de biens et d'humains, ainsi que le développement sans précédent d'une culture du divertissement de masse, concourt à la nervosité des corps toujours plus sollicités. Le cinéma vient, dès le début du $\mathrm{XX}^{\mathrm{e}}$ siècle, s'ajouter au catalogue des agents pathogènes du système nerveux, jouxtant la litté-

\footnotetext{
5 Brochin 1878, 360.

6 Bouveret 1891, 41.

7 Bouveret 1891, 11.

8 Bouveret 1891, 11sq.

9 Borel 1894, 4sq.

10 Borel 1894, 36.

11 Brochin 1878, 362.

12 Bouveret 1891, 17 .

13 Bouveret 1891, 25.
} 
rature populaire accusée de déchaîner dépravation mentale et somatique, comme en atteste le professeur d'hygiène à Berne, le Dr Girard, qui vilipende «la littérature immorale, des images obscènes, des spectacles inconvenants» ${ }^{14}$. Ainsi, le nervosisme infère chez le sujet une sursensibilité éveillée en permanence par le moindre bruit, image, scène ou émotion dont la vivacité peut aboutir à une altération sérieuse des facultés sensitives.

L'analyse d'un corpus constitué autour des questions qui agitent les milieux médicaux et paramédicaux du tournant du $\mathrm{XX}^{\mathrm{e}}$ siècle permet de mettre au jour la manière dont le cinéma est reçu par les savants et les observateurs qui questionnent son statut dans la création ou l'aggravation de maux souvent perçus comme des dommages collatéraux de la modernité. Marqué par des spasmes, des stases ou des hallucinations, le corps névrosé renvoie à une symptomatologie voisine du diagnostic posé sur les spectateurs affectés par le «mouvement vibratoire ${ }^{15}$ d'une représentation vécue sur le mode traumatique. Telle est la formule employée par le Dr Lojacono, psychiatre et professeur de l'Université de Palerme, qui rapporte en 1912 deux cas cliniques révélant «l'action dommageable des projections cinématographiques sur les personnes nerveuses ${ }^{16}$. Parmi les facteurs incommodants, il consigne: l'obscurité de la salle, les turbulences de la mécanique projective qui porte atteinte autant à la vision qu'à l'ouïe, et les images «tragiques» du film. Instiguant crises de panique, insomnies, phobies et/ou délires hallucinatoires, la vision filmique précipite et multiplie les symptômes de personnes déjà fragilisées par la neurasthénie ou l'alcoolisme. Lojacono insiste sur la virulence des impressions laissées par les images sur le spectateur qui ne cesse d'être assailli, suite à la projection, par des visions et des hallucinations diurnes et hypnagogiques dupliquant les scènes à l'origine du mal.

Cette connivence entre malaise psychique et dispositifs audiovisuels semble confirmée par les discours alarmistes portés à l'endroit du cinéma par les ligues d'hygiénisme, à l'exemple des articles du pasteur zurichois A. Wild publiés dans les Annales suisses d'hygiène scolaire ${ }^{17}$. De 1911 à 1916, ce dernier s'engage très activement dans ce qu'il nomme la «lutte contre le cinéma d'infestation», passant en revue toutes les prescriptions légales en vigueur dans chaque canton, ainsi qu'en Europe. Il semblerait, en effet, qu'il faille attendre les années 1910 pour que les personnes soucieuses de prophylaxie sociale et mentale prennent la mesure des nuisances causées par le cinéma, exigeant des autorités des décrets officiels pour les endiguer. Discutant des

14 Girard 1900, 44.

15 Lojacono 1912.

16 Lojacono 1912, 14.

17 Wild 1911-1916. Wild emploie les termes de «Nervenerschütterung» (ébranlement nerveux) et de «Nervenüberreizung» (surexcitation nerveuse) (Wild 1911, 49). 
répercussions de ce préjudice moderne, Wild estime qu'une partie des jeunes délinquants est influencée de manière significative par des films qui, en flattant leurs bas instincts, servent surtout à faire frissonner les spectateurs. Il cite abondamment des voix autorisées, notamment dans le champ du corps médical, qui mettent en garde contre les films sensationnalistes au moralisme douteux, mais aussi contre la surexcitation, l'ébranlement nerveux et l'excitation de la circulation sanguine entraînée par un spectacle qui «empoisonne l'imagination et ruine les nerfs $»^{18}$. Un auteur cité par le pasteur relève la charge malfaisante des films à trucs dont les scènes à transformation consistent en une substitution effrénée d'images et de métamorphoses dont la valeur éthique et esthétique frise le néant. Si le contenu des images apparaît comme la cible favorite des réformateurs sociaux qui craignent des épidémies de petite ou grande criminalité, le principe de projection d'une multitude de photogrammes sur un écran lumineux - avec ses corollaires de scintillement, de vibration et de vacillement du perçu - soulèvent la peur d'une irritation du système nerveux ${ }^{19}$. En 1915, deux psychiatres italiens, Masini et Vidoni ${ }^{20}$, avancent l'hypothèse d'une «psychose spéciale» due au cinématographe qui encourage les «impressions délétères du faux réalisme ${ }^{21}$, des hallucinations visuelles à caractère «vivace, net et précis», et des dérangements sensoriels liés au passage effréné des images sur la toile. Proposant d'interdire l'accès au cinéma aux malades nerveux, ils lisent l'émoi suscité par la séance comme génératrice de «convulsions, terreurs nocturnes, névroses, troubles oculaires, etc.», mais aussi d'«états d'angoisse, hallucinations et idées délirantes $»^{22}$. Se référant à des enquêtes accréditées, ils confirment l'existence de crimes commis sous la suggestion d'images cinématographiques, rapprochant ces délits d'une forme de criminalité dite «épileptique» en raison du caractère mécanique et foudroyant d'un acte exécuté sous l'impulsion d'une hallucination à teneur agressive ${ }^{23}$. Conjuguant le paradigme de l'excitabilité sensori-motrice et de l'anesthésie morale, ces descriptions indiquent combien le cinéma peut tout à la fois traumatiser et hanter l'esprit et le corps du spectateur.

Porte-parole des adversaires du cinéma, Louis Haugmard l'assigne à une distraction pour «l'imagination» et la «sensibilité populaires, déprimées et

18 Wild 1912, 70.

19 Wild 1912, 86. Il précise que si le cinéma porte atteinte aux âmes, il a des conséquences physiques sous la forme d'une agitation et d'une irritation des nerfs, mais aussi d'une excitation et d'une blessure des yeux.

20 Masini/Vidoni 1915.

21 Masini/Vidoni 1915, 621.

22 Masini/Vidoni 1915, 620.

23 Masini/Vidoni 1915, 623. 
lasses», octroyant «à nous les sédentaires et les énervés de participer à des sensations héroïques» ${ }^{24}$. Affirmant le «pouvoir infiniment dangereux qui est le sien $[\ldots]$ pour nourrir toutes les vanités et déclencher les imitations, car l'image est excitatrice», le chroniqueur du Correspondant prophétise l'ère des «foules charmées» dont la raison s'atrophiera progressivement dans l'exercice régulier d'un agrément aussi futile qu'abrutissant. «Le cinématographe», conclut-il, «sera l'<amphithéâtre〉 des civilisés affaiblis, les 〈circenses〉 pour peuple pacifiste, de l'action pour neurasthéniques $»^{25}$. Entrelaçant représentation et spectateur dans une structure mimétique, cet argumentaire fin de siècle qualifie de névrosés les consommateurs du cinématographe qui ne font que péjorer leur déviance, amplifiant du même coup le contingent de sujets amorphes déjà accouchés par une civilisation mise en péril par le spectre de la contagion mentale. Dans une optique semblable, certains commentateurs du cinématographe rapprochent les convulsions du corps hystéro-épileptique de celles subies par le spectateur de cinéma emporté par la vision d'une image grisante et galvanisante, d'une vision donc qui opère sur lui sur le mode d'un courant électrique. L'imaginaire électrique contamine d'ailleurs l'ensemble des paramètres constitutifs de la séance cinématographique dont l'image est réputée transmettre au sujet percevant une pulsion qui le pousse à mimer les trépidations perçues à l'écran. Ces thèses sur l'instinct imitatif s'appuient sur les acquis de la psychophysiologie qui pose que chaque perception ou sensation s'accompagne irrémédiablement d'un mouvement inconscient, intérieur ou non ${ }^{26}$. Le cinéma réveille précisément chez le sujet son «inconscient cérébral»», à savoir des automatismes et des réflexes inscrits dans les couches les plus primitives de son système nerveux. L’image cinéma qui défile «frénétiquement» et le jeu «hystérique» des

\section{Haugmard 1913, 766sq.}

25 Haugmard 1913, 771.

26 C'est notamment la thèse de Charles Féré qui l'érige en présupposé de la psychologie moderne: «L'existence d'une relation nécessaire entre le mouvement et toute sensation ou toute représentation mentale, propre à établir que toutes les opérations psychiques ont nécessairement un équivalent moteur, constitue une notion très importante en psychologie, et cette notion peut être immédiatement utilisée par l'interprétation d'un phénomène qui a beaucoup attiré l'attention dans ces dernières années, je veux parler de la suggestion mentale dans la communication de la pensée» (Féré 1887,116).

27 Gauchet 1992. Les historiens des sciences du psychisme distinguent deux conceptions de l'inconscient: une conception physiologique qui met l'accent sur la nature réflexe et mécanique du système nerveux, et qui est défendue par des savants britanniques et allemands, ainsi que par Hippolyte Taine ou Alfred Maury; et une conception psychique qui, sans rejeter complètement le modèle mécaniciste, octroie au subconscient un pouvoir de créativité échappant au déterminisme automatiste, comme l'illustrent les travaux de Pierre Janet en France. Ces deux acceptations de l'inconscient sont toutefois tributaires du même schème évolutionniste qui envisage les faits et entités psychiques comme gradués en fonction d'une échelle marquant leur perfectibilité. Voir à ce sujet Richard 2008. 
acteurs présentent une nocivité pour le sujet dont on souligne le gradient d'excitabilité électrique, le réduisant à un pur conduit traversé par des influx émanant d'une source «magnétisante». Le spectateur de cinéma rejoint ainsi la cohorte des «tempéramentsélectriques $»^{28}$ observables dans la population des névrosés et autres natures suggestionnables.

\section{Le sujet paramnésique}

Les troubles de la mémoire permettent d'envisager un mode d'articulation supplémentaire entre le sujet morbide et le sujet cinématographique, notamment grâce à des occurrences où le cinéma fournit un outil épistémologique renseignant sur le fonctionnement de l'appareil psychique. Autour de 1900, l'idée d'images latentes et virtuelles est tout spécialement mobilisée dans des essais portant sur les «exaltations pathologiques de la mémoire», comme dans le cas du phénomène dit du «Moi des mourants» qui se produit lorsqu'une personne échappe de peu à la mort et revoit avec une très grande précision les scènes prégnantes de son existence. En 1896, le philosophe Victor Egger rapporte le témoignage d'un savant suisse, le professeur Heim, dont l'âme a revu «tout le cours de sa vie passée» pendant une chute durant une excursion en montagnes. Précisant l'«extrême rapidité de la pensée et de l'imagination», il poursuit ainsi:

Ce que j'éprouvai [...] durant les quelques secondes de ma chute, il me faudrait maintenant une heure pour le raconter; toutes les pensées et toutes les images s'offraient à moi avec une précision et une clarté extraordinaires. [...] Ensuite j'aperçus tous les faits de ma vie passée se déroulant devant moi en d'innombrables images. ${ }^{29}$

Cette «succession rapide de tableaux à chacun desquels correspond une émotion» est justifiée par Egger par le «sentiment synthétique de la vie passée» que la mort inspire puisqu'elle impose «l'arrêt brusque de la série des états de conscience conservés dans la mémoire» ${ }^{30}$. Si le moi des morts rapides s'exprime «tout en images» ${ }^{31}$, c'est en raison de la soudaineté des événements qui accule le psychisme au langage visuel, plutôt que verbal ou abstrait. Le

28 Le docteur Louis Tollemer, dans une analyse des chocs causés par les accidents électriques, estime que «tous les hommes eux-mêmes ne sont pas également sensibles aux chocs électriques, et on peut aussi admettre, pour chaque individu, des variations de la susceptibilité électrique». Si la moiteur de la peau, son épaisseur, le parcours suivi par la décharge, la surface de contact jouent un rôle important dans la blessure, «le tempérament nerveux, l'état de santé ou de maladie, l'état d'ivresse ou de sobriété, l'état de sommeil ou de veille» sont aussi à prendre en compte. (Tollemer 1902, 546; M. B. souligne.)

29 Egger 1896, 27.

30 Egger 1896, 28sq.

31 Egger 1896, 30. 
moi des mourants est donc compris comme un mouvement régrédient vers un état reculé du développement mental où l'image prime sur le verbe, où l'irrationnel l'emporte sur le rationnel. Ce n'est donc pas un hasard si les figurations de la mort imminente sont systématiquement étayées sur des dispositifs d'images animées, comme le panorama mobile ou le cinématographe qui déploient une consécution d'images signalées tantôt comme tableaux, tantôt comme photographies, tantôt comme toile peinte qui se déroule verticalement. Notons à cet égard que si le recours à un dispositif visuel semble privilégié pour ses vertus didactiques, il procure surtout le moyen de traduire la temporalité en termes d'espace, à l'instar du Freud de L'Interprétation des rêves qui conçoit l'appareil psychique comme un instrument optique au sein duquel transite une énergie psychique sectoriellement orientée, l'histoire du sujet se dépliant à même un lieu cependant anatomiquement introuvable. Le sujet voit donc le temps (de son histoire) défiler à l'intérieur d'un espace accueillant celle-ci sous la forme de représentations audiovisuelles.

La paramnésie, ou l'illusion du déjà vu et vécu, convoque également une série de représentations mettant en jeu des qualités formelles et des états subjectifs référables à la projection d'images animées. En 1905, les docteurs Dromard et Albès publient un essai théorique sur l'expérience de «l'illusion dite de fausse reconnaissance», appelée ailleurs sensation de déjà vu ${ }^{32}$. Transcrit sous les allures d'une activité spectatorielle, cet état mental particulier n'est pas sans rappeler le fonctionnement du cinéma qui induit chez le sujet percevant un processus de dédoublement du moi en une instance agissante (qui s'assimile à l'imaginaire filmique) et une instance observatrice (qui reste à distance de l'écran). Tel est le compte-rendu autobiographique de l'un des auteurs dont l'illusion de fausse reconnaissance a été déclenchée par une «éclipse totale de tout travail psychique en vue du réel»:

$\mathrm{Au}$ début, ma personnalité semble s'isoler du monde extérieur, et comme se détacher de l'ambiance. Tout ce qui m'entoure me paraît de plus en plus lointain et comme suspendu dans le vide. La vie flotte en dehors de moi, et toutes les sensations qu'elle m'apporte défilent indifférentes et sur un même plan, comme des ombres chinoises qu'un impalpable rideau séparerait de mon contact. Ces sensations, d'ailleurs, il me semble qu'elles viennent à moi plus que je ne vais à elles, je les subis plutôt que je les prends et je n'ai en aucune façon le sentiment d'un travail actif en vue de les faire miennes. Puis petit à petit, je rentre en moimême; je me regarde voir, je m'écoute entendre. Il me semble alors que je suis à la fois deux hommes dont l'un fonctionne en automate et dont l'autre regarde fonctionner le précédent, celui-ci assistant à tout ce qu'éprouve celui-là, comme un spectateur désintéressé. [...] L'illusion est intégrale: je veux dire que le sentiment que j'éprouve ne répond pas à une simple analogie mais à une identité parfaite. Ici [...], j'ai l'impression que la réalité présente a son double, mais ce double, je n'ai pas plus de raison de le placer dans le passé que dans l'avenir. [...] Un double virtuel semble accompagner leur réalité, mais ce double virtuel je ne saurais dire exactement si je dois l'appeler souvenir et non pas aussi bien une prévision. ${ }^{33}$

32 Dromard/Albès 1905.

33 Dromard/Albès 1905, 217. 
Cette description rassemble plusieurs attributs et processus psychiques manifestes dans les crises hystériques, épileptiques ou somnambuliques qui s'accompagnent souvent d'un dédoublement intrapsychique affectant la réalité d'une perception toujours escortée d'un double virtuel situé, ici, dans une temporalité hétérogène. Le récit met en évidence cette scission du moi en deux instances complémentaires qui se distribuent, de part et d'autre du «voile» métaphorique, en une fonction opérante et une fonction réceptrice, l'une étant le spectacle de l'autre. A l'instar de l'écran cinématographique, le voile des ombres chinoises sert de support bidimensionnel à un défilé d'images et de sensations que le sujet subit sans en avoir la maîtrise. Réservoir passif de stimuli en provenance d'un lieu situé à l'extérieur de soi, ce spectateur s'incorpore alors littéralement en lui-même, effaçant la frontière qui séparait ces deux espaces-temps délimités comme appartenant, l'un à l'ordre du rêve, l'autre à l'ordre de la réalité. Le recours au paradigme photographique - récurrent dans les théories de la mémoire et de ses diverses pathologies ${ }^{34}$ - enseigne comment le sujet réactive une image sauvegardée en mémoire, l'impression de déjà vu semblant reproduire dans la positivité de la vie consciente une «tranche de vie» passée. Plus loin, dans leur tentative d'explication rationnelle de cette anomalie, les auteurs reviennent sur l'idée de la mémoire comme espace de stockage d'images, cet emmagasinage «dans l'obscurité du subconscient $»^{35}$ opérant à l'insu du sujet ${ }^{36}$. Si tout le vocabulaire mobilisé renvoie à la photographie - réalisme, empreinte, double, virtuel, net -, ces notions peuvent aussi s'étendre à la représentation cinématographique qui confère à cette perception un mouvement par ailleurs également évoqué dans les phénomènes paramnésiques. Dans tous les cas, le sujet percevant devient le site où ressurgissent des images psychiques attribuées à une autorité extrinsèque, produisant une sensation proche de l'inquiétante étrangeté freudienne qui rend notamment compte des effets perturbants du double et du reflet. Passant en revue diverses théories, Dromard et Albès optent pour l'hypothèse d'un dysfonctionnement du «stéréoscope intérieur» ${ }^{37}$ qui ne parvient pas à superposer les deux images

34 Voir par exemple les thèses de Théodule Ribot (1897 [1881]).

35 Dromard/Albès 1905, 221.

36 Ils poursuivent ainsi leur démonstration: «Les centres psychiques, nous dit-on, peuvent recueillir des impressions sans état de conscience, et il arrive ainsi que nous découvrons en nous-mêmes des images sans pouvoir saisir le moment ni le mode d'acquisition de ces dernières. Une représentation ayant été emmagasinée dans la mémoire des centres polygonaux sans intervention du centre 0 , on peut voir cette représentation surgir un beau jour de leurs profondeurs, par un phénomène de recollection, et sous l'influence d'une réalité», (Dromard/Albès 1905, 220).

37 Dromard/Albès 1905, 222. 
déposées dans l'esprit - la perception de tout objet se faisant toujours à double -, la copie émoussée de l'image originale étant associée (à tort) à un vécu antécédent ${ }^{38}$. Ainsi, «les deux images ne se confondent plus de manière à ne représenter qu'un objet», mais «par une sorte de 〈mirage> la plus faible serait projetée dans le passé» ${ }^{39}$. La mention du jouet optique permet ainsi de concevoir le sujet comme une sorte d'appareil actualisant une disjonction temporelle qui recoupe une disjonction psychique responsable du sentiment contradictoire d'être à la fois sujet et objet de la perception. Dans tous les cas, la paramnésie limite la perception à un fonctionnement machinal et spontané, l'introspection du sujet qui se voit regarder le monde qui l'entoure étant interprété comme une expression rudimentaire et aberrante de l'attention.

Le spectateur de cinéma apparaît également dans les discours comme un récepteur assujetti à l'écoulement irrésistible du film ou comme un rêveur plongé dans l'illusion de réalité du perçu, sa subjectivité étant partagée entre deux phénoménalités, l'ici de la salle de projection et l'ailleurs de l'univers diégétique, le présent de l'acte perceptif et le passé de la prise de vue, l'inertie du corps actuel et la versatilité de l'imaginaire filmique. De manière générale, tous les états mnésiques «anormaux» - que la mémoire fasse défaut, fasse excès, fasse surimpression ou fondu enchaîné - dressent le portrait d'un sujet aux prises avec un passé ravivé sur le mode de la projection d'images animées ressenties sur le mode traumatique. Cette figure du «film intérieur» réapparaît dans la littérature médicale à propos de la fragilisation des sujets vulnérables (malades, enfants, adolescents, femmes) qui, en se rendant au cinéma, s'exposent au risque de vivre la séance comme un instant pénible renforçant leurs symptômes ou leur impressionnabilité constitutive. Certains fragments du film sont alors «répétés» mentalement dans un aprèscoup qui transforme le film vu en film vécu et revu dans la souffrance. En effet, un grand nombre de patients qui témoignent de leurs expériences au cinématographe ne cessent de revoir les scènes prégnantes du film sous la forme d'hallucinations diurnes ou nocturnes qui s'étalent devant les «yeux

38 On décèle ici l'influence d'Hippolyte Taine qui explique comment chaque sensation (ou perception) imprime dans l'esprit (la mémoire) un substitut (une image) qui peut renaître spontanément: «Voilà un second groupe de sensations, si semblables aux premières qu'on peut les appeler sensations reviviscentes, et qui répètent les premières, comme une copie répète un original ou comme un écho répète un son. A ce titre, elles ont les propriétés des premières, elles les remplacent en leur absence, et, faisant le même office, elles doivent donner lieu au même travail mental» (Taine 1883,15). C'est ce qui l'amène à penser que la perception correspond à une sorte d'hallucination vraie, «au moins à l'état naissant». Car «l'image, répétition spontanée de la sensation tend comme elle à provoquer une hallucination» (Taine 1883, 24).

39 Les auteurs mentionnent la thèse de Fouillée (1885) qui «admet que toute sensation nouvelle s'accompagne d'un retentissement, d'une sorte d'écho, qui la répète en la fixant dans la masse de nos connaissances» (Dromard/Albès 1905, 222). 
de leur esprit» - selon la locution de Macario $^{40}$ - comme si elles étaient projetées sur leur écran psychique. Qu'elles soient définies comme des visions, des impressions hypnagogiques ou des hallucinations, les images filmiques reviennent hanter le malade qui performe machinalement la représentation gravée dans son subconscient.

\section{Le sujet somnambulique}

C'est sur le terrain de la compulsion à la répétition et de la hantise que semblent se rejoindre les expressions extravagantes de la mémoire et les phénomènes liés à l'hypnose où prédominent les personnalités doubles et multiples. Le modèle des sciences du psychisme prolifère parfois sous la forme d'une subjectivité possédée par une puissance extérieure, par une personnalité parallèle qui prend le dessus sur le Moi conscient pour mener une vie concurrente. Le thème de la métamorphose et de l'arborescence subjective, un seul individu pouvant «proliférer» dans diverses personnalités partiellement indépendantes du sujet-matrice, forme le noyau des transes médiumniques vécues par Mlle Hélène Smith dont le médecin et philosophe des sciences genevois Théodore Flournoy a rendu compte dans son ouvrage Des Indes à la Planète Mars. Etude sur un cas de somnambulisme avec glossolalie $(1900)^{41}$. Dans cette étude de cas, Flournoy donne des facultés supposément paranormales d'Hélène Smith une interprétation rationnelle sur la base d'une anamnèse psychopathologique mettant en relief l'extrême influençabilité d'un sujet traversant régulièrement des états hypnoïdes qui vont favoriser l'émergence de romans somnambuliques se subdivisant en un cycle martien, un cycle hindou et un cycle royal. Chacun de ces cycles se rattache à une personnalité subconsciente où la médium joue, successivement, le rôle d'ambassadrice de la planète Mars, de princesse hindoue et de Marie-Antoinette, ces voyages dans le temps et dans l'espace provoquant l'invention et l'usage d'une langue inédite (glossolalie), la langue martienne, liée au périple astronomique.

Le somnambulisme permet de rapprocher le cinéma et les sciences du psychisme, non seulement sur la base du motif du Moi dissocié, le spectateur incarnant une sorte d'automate obéissant à sa conscience subliminale (sub-

40 Macario 1857.

41 Flournoy 1900. Pour une analyse détaillée du rôle de Flournoy dans l'histoire des sciences du psychisme, je renvoie aux travaux de Vincent Barras qui a étudié son apport aux théories de l'inconscient conçu comme un moi subliminal particulièrement sensible aux inductions extérieures. Voir par exemple Barras/Vidal 1996. 
consciente), mais aussi sur les modalités qui régissent ce dédoublement, le sujet en transe se transformant en appareil à projeter des images en mouvement. Flournoy propose d'ailleurs le néologisme d'«automatiste» ${ }^{42}$ pour remplacer le terme antiscientifique de médium employé par les partisans du spiritisme, insistant ainsi sur la dimension irréfléchie et involontaire de pratiques qui ne doivent rien à l'intervention d'un esprit de l'au-delà, mais tout à l'excentricité d'un esprit frappé de désagrégation mentale. Soulignant sa tendance marquée à la rêvasserie et au «vagabondage de la pensée» ${ }^{43}$ (auquel la femme serait naturellement encline), le savant explique que «ses visions sont accompagnées d'un certain degré d'obnubilation» la rendant «inerte et passive, souvent avec une nuance de béatitude et de bien-être extatique, devant le spectacle qui s'offre à elle» ${ }^{44}$.

Le texte de Flournoy contient, en effet, de nombreux passages qui décrivent la médium comme spectatrice dont le regard s'absorbe dans les «visions se déroulant devant son regard éveillé», lui prodiguant «un spectacle inattendu et toujours renouvelé qui faisaient de ces séances [de spiritisme] une partie de plaisir». Ce plaisir est dû en effet à l'attraction formidable que la médium exerce sur son public d'adeptes, car comme l'écrit Flournoy, «les scènes de somnambulisme et d'incarnations avec leurs phénomènes physiologiques divers, catalepsie, léthargie, contractures, [...] ajoutent une grande variété et un puissant intérêt de plus aux très remarquables et instructives productions médiumniques d'Hélène Smith $»^{45}$. A elle toute seule, donc, elle semble fournir les conditions d'un spectacle audiovisuel intense se développant dans une semi-obscurité nécessaire aux apparitions sublimino-spectrales, un spectacle donc qui se joue à la fois au-dedans et au-dehors d'un corps qui reçoit, produit et restitue les impressions suscitées par les transes. A l'instar de l'appareil de prise de vue Lumière inventé l'année même où se magnifie la fantasmagorie de ces fictions somnambuliques - une machine donc dotée de fonctions réversibles de captation et de projection d'images -, Hélène Smith fabrique, enregistre et projette le fruit d'un travail subconscient qu'elle attribue à des manifestations suprasensibles dont elle se croit être le simple réceptacle, cette passivité étant partiellement reconnue par Flournoy, bien qu'interprétée en des termes objectifs qui expurgent le surnaturel au profit du constat de sensitivité d'un sujet victime de ses facultés inférieures.

42 Flournoy 1900, XI.

43 Flournoy 1900, 17.

44 Flournoy 1900, 51.

45 Flournoy 1900, 7. 
Apparaissant dans le riche répertoire de Georges Méliès qui reprend à son compte, tout en les détournant par le biais du comique, les angoisses liées à la démultiplication des corps et des esprits, cette hantise de la scission intrapsychique touche également les discours sur le cinéma qui se préoccupent de ses effets hypnotiques ${ }^{46}$. La figure du double moi, centrale aux études sur les névroses, sur la paramnésie, autant qu'aux recherches psychistes, fait apparaître un modèle de subjectivité où deux consciences sont mises en relation dans une spécularité et une spectactularité, où l'une devient le reflet et le champ d'observation de l'autre, la première se mettant en scène pour la seconde, clôturant ainsi l'économie visuelle dans une logique schizoïde. Ce dispositif regardé/regardant impliquant une dissociation mentale forte par laquelle le sujet devient l'objet de son propre regard, dépossédant du même coup le corps médical d'une partie de ses prérogatives, ce dispositif donc investit régulièrement les différents formations discursives analysées, témoignant de l'ample diffusion de la théorie janétienne du subconscient automatique comme forme élémentaire de la conscience supérieure ${ }^{47}$. Fournissant une allégorie du dispositif cinématographique, les conduites réflexes du double moi peuvent ainsi être mises en rapport avec les représentations du spectateur de cinéma comme sujet conditionné, à l'intérieur de lui-même, par des injonctions ressenties comme allogènes.

\section{Le cinéma: un laboratoire expérimental du sujet moderne?}

Au carrefour du corps et de l'esprit, les pathologies psychophysiologiques ménagent un champ d'observation privilégié à la médecine qui s'interroge sur les causes entraînant les désordres psychiques. Très souvent conceptualisé en termes de réceptivité et de réactivité accrues, le sujet percevant va faire l'objet d'une littérature qui s'emploie à élucider l'origine de nouvelles maladies référées à la modernité et à ses progrès technologiques, scientifiques et culturels. Minorant l'argument incantatoire sur l'hérédité des tares, un nombre appréciable d'étiologies prennent en compte une série d'autres facteurs, dont celui du milieu environnemental et social. Parmi ces coefficients générateurs de subjectivités «insolites», le cinéma est souvent jugé, implicitement ou explicitement, comme responsable d'occasionner une modification de la configuration psychique dont la «modernisation» est perçue, soit comme une adaptation nécessaire à son biotope, soit comme une dégé-

46 Hellwig 1916.

47 Janet 1898. Les phénomènes d'autoscopie, courants chez les hystériques, participent du même schème. 
nérescence regrettable. Au-delà des divergences d'opinions sur la nature du cinéma, je propose d'envisager le spectateur comme une émanation de la modernité urbaine qui crée des individus en quête de sensations fortes exerçant alternativement des effets tonifiants et débilitants, fortifiants et relaxants, l'excitation du système nerveux suivant des courbes cyclothymiques qui réclameront des remèdes antithétiques ${ }^{48}$.

Certains discours réactionnaires permettent de jauger des répercussions de cette «nouvelle» technologie qui est déchiffrée comme un signe de décadence culturelle. Ainsi, considéré comme le «théâtre des illettrés» ${ }^{49}$ par René Doumic, le cinématographe n'est qu'un préjudice autant du goût esthétique que de la physiologie du spectateur qui se voit confronté à une image tremblotante dont les fébriles protagonistes semblent frappés du même fléau que les patients neurasthéniques:

Vous vous asseyez, vos yeux s'écarquillent, et ce qu'ils perçoivent d'abord, sur l'écran lumi-
neux dont la surface éclairée perce seule toute cette ombre, c'est un tremblement continu.
Cela vibre, cela vacille, cela trépide, cela ne s'arrête pas de trembler. Encore un effort d'ac-
commodation. [...] Formes sans consistance de corps, sans épaisseur, comme on n'en voit
qu'en rêve. [...] Images impalpables et floues, tout à la fois réalistes et irréelles. Ces appa-
ritions vont et viennent, comme nous faisons nous-mêmes, quand nous sommes très agités.
On a dans ce monde blafard une démarche raide comme frappée d'ankylose, avec des gestes
d'automates. [...] Les visages expriment une surprise violente ou un violent désespoir, la joie,
la douleur, la colère, mais toujours à l'état violent. [...] Il arrive qu'ils soient à bicyclette ou
en automobile, deux genres de sports qui sont très répandus parmi eux. Tout à coup, sans
qu'on puisse deviner pourquoi, et comme s'ils étaient pris d'une subite frénésie, ils accélèrent
le mouvement, ils le précipitent, ils vont sortir du cadre et foncer sur nous».0

Ce texte parcourt l'ensemble des thématiques dégagées par notre croisement entre cinéma et sciences du psychisme: il est question de trépidation, d'illusion de réalité, d'évanescence, d'agitation, d'atonie, d'automatisme, de vitesse, de heurt, etc., et ceci, aussi bien dans l'image que dans le corps des sujets percevants. Résolument moderne, cette esthétique du choc et de la saccade sera tantôt condamnée par les intellectuels refusant d'annexer le cinéma au panthéon des arts nobles, tantôt valorisée par les partisans des avant-gardes artistiques qui célèbrent la spécificité d'une forme d'expression révolutionnaire et démocratique. Quoi qu'il en soit, le cinéma postule un type de sujet qui se voit bousculé, interpellé et captivé par une représentation qui en appelle très directement à ses impressions cénesthésiques, lui assignant une posture menant à une fragmentation possible de sa subjectivité.

48 Les médecins mettent en exergue la périodicité des fonctions et des opérations psychiques dans les maladies mentales comme la neurasthénie. Voir par exemple: Pailhas d'Albi 1910. C'est pourquoi les remèdes prescrits préconisent, tantôt le repos complet dans les phases de surexcitation, tantôt la stimulation dans les moments de torpeur.

49 Doumic 1913, 930.

50 Doumic 1913, 920sq.; M. B. souligne. 
Or, autour de 1900, les sciences du psychisme élaborent des modèles de subjectivité témoignant d'une crise plus large du moi envisagé comme une entité autonome et maîtrisable. La psychologie expérimentale et psychopathologique, notamment, formalise un spécimen humain à partir de l'étude des différents états traversés durant des épisodes maladifs, débouchant parfois sur une simulation de la folie vouée à fournir une herméneutique du sujet bien portant. Dans les laboratoires de psychologie appliquée, on lui fait subir fatigues, secousses, transes, parésies, perceptions extra-sensorielles, etc. afin d'en tirer les lois générales d'une subjectivité normale et normée. Car, à travers ces multiples détours par des stratégies de perturbation de la mécanique nerveuse, c'est une psychologie à validité universelle qui est ambitionnée ${ }^{51}$. Paradoxalement, cette visée positiviste s'attache essentiellement à un sujet atypique et extraordinaire, un sujet qui conjoint des aspects pré-modernes et modernes, c'est-à-dire qui combine des phénomènes pluriséculaires (vision, ravissement, divination, télépsychie, etc.) à des tourments originaux (chocs électriques, lésions oculaires et auditives ${ }^{52}$, ennui moderne ${ }^{53}$, phobies sociales, psychonévroses ferroviaires, etc.). Cet assemblage d'archaïsme et de contemporanéité se vérifie dans le cadre du paradigme de l'hypnose qui réadapte la tradition du sujet intransé à un contexte où les dispositifs magnétiques se technologisent via, par exemple, le couplage à des appareils de mesure ou des spéculations théoriques dévolues à objectiver les données fugitives et impalpables de l'esprit ${ }^{54}$. Le sujet de la modernité est ainsi issu d'efforts pour contrôler une combinaison curieuse de faits anciens et nouveaux qui excède le champ de compétences des instances savantes et dirigeantes, laissant affleurer des inquiétudes à propos de l'intégrité d'une conscience aux manifestations aléatoires et, notamment, automatiques. Modélisées à l'image des technologies de la modernité (photographie, phonographe, télégraphe, cinématographe, etc.), les théories de la subjectivité en 1900 enregistrent les potentialités épistémiques d'instruments qui, tout en prolongeant prothétiquement les fonctions perceptives de l'appareil psychique, soulèvent des anxiétés quant à l'indépendance d'un sujet

51 Les travaux de Josefa Joteyko sur la fatigue, menés au sein du Laboratoire Kasimir de l'Université de Bruxelles, sont exemplaires en la matière.

52 A l'instar de la «cinématophtalmie» causée par la consommation assidue de films. Ginestous 1909.

53 Tardieu 1903.

54 Au sein du champ de l'hypnose, cette objectivation du subjectif se vérifie à plusieurs niveaux: sur un plan «concret», d'abord, à travers la mise au point d'appareils de mesure comme l'hypnoscope d'Alexandre Baréty (Baréty 1887); au niveau de l'association entre imaginaire hypnotique et technologie moderne à l'œuvre dans les recherches sur l'«hypnotisme téléphonique» (Bérillon 1912); au niveau de la supputation théorique avec les travaux de Jules Luys sur la couronne magique (Luys 1894). 
aux prises avec le monde machinique et industriel. Le sujet automatique du somnambulisme, de la névrose ou de la paramnésie apparaît donc comme, simultanément, le symptôme, le symbole et la critique d'une modernité qui environne les sciences du psychisme, les mettant en rapport dialogique avec le monde des arts et des spectacles.

Ce sujet scientifique créé en laboratoire présente, en effet, un certain nombre de points de contact avec le spectateur de cinéma, permettant d'avancer l'hypothèse d'un passage de l'un à l'autre sous la forme d'un relais pris par le cinéma au moment où la psychologie abandonne le terrain du merveilleux scientifique pour investir des objets autrement plus solides et moins controversés que ceux offerts par l'hypnose ou l'hystérie ${ }^{55}$. Le sujet cinéma pourrait se révéler en tant que prototype du sujet «extraordinaire» des sciences du psychisme, un sujet divisé, magnétique et fasciné, mais aussi un sujet moderne ravivant un imaginaire archaïque. Partant, le spectateur auquel s'adresse le cinématographe à ses débuts est un sujet qui lui préexiste, qui est préparé à l'accueillir et qui peut même être considéré comme une variante possible du sujet expérimental, un sujet pur produit de la science et de la culture de cette fin de siècle. Si cette recherche consiste à se demander si le spectateur de cinéma n'indique pas la voie vers la construction d'un sujet expérimental de la modernité, cette question en soulève une autre, plus vertigineuse et osée encore, celle de la disparition du sujet au profit d'une production mécanisée des corps fabriqués à la chaîne par la société de consommation du $\mathrm{XX}^{\mathrm{e}}$ siècle. Car rien de plus versatile et fragile, en effet, que cette subjectivité dont l'indépendance et l'homogénéité semblent sans cesse menacées d'éclatement par un milieu potentiellement hostile, bien qu'au service d'un progrès technologique censé apporter bien-être individuel et prospérité collective.

\section{Bibliographie}

Albera, François/Maria Tortajada, «L'Epistémè 〈1900»», dans: A. Gaudreault/C. Russell/P. Véronneau (sous la dir.), Le Cinéma, nouvelle technologie du XX siècle (Lausanne 2004) 45-62

Baréty, Alexandre, Le Magnétisme animal, étudié sous le nom de force neurique rayonnante et circulante, dans ses propriétés physiques, physiologiques et thérapeutiques (Paris 1887)

Barras, Vincent/Fernando Vidal, «La Suisse romande <à la découverte de l'inconscient»», Revue médicale de la Suisse romande 116 (1996) 909-915

Bérillon, Edgar, «Hypnotisme téléphonique», Revue de psychothérapie et de psychologie appliquée 2 (août 1912) 62-63

55 Cette expression de merveilleux scientifique est empruntée à Durand de Gros (1894), et a été analysée par Régine Plas (2000). 
Borel, Virgile, Nervosisme ou neurasthénie. La maladie du siècle et les divers moyens de la combattre (Lausanne 1894 [1873])

Bouveret, Léon, La Neurasthénie. Epuisement nerveux (Paris 1891)

Brochin, Hippolyte, «Maladies nerveuses», Dictionnaire encyclopédique des sciences médicales (Paris 1878) 332-391

Doumic, René, «L'Age du cinéma», Revue des Deux Mondes 16 (1913) 919-930

Dromard, Gabriel/Dr Albès, «Essai théorique sur l'illusion dite de 〈fausse reconnaissance»», Journal de psychologie normale et pathologique (1905) 216-228

Durand de Gros, Joseph-Pierre, Le Merveilleux scientifique (Paris 1894)

Egger, Victor, «Le Moi des Mourants», Revue philosophique XLI (janvier-juin 1896) 26-38

Féré, Charles, Sensation et mouvement: études expérimentales de psycho-mécanique (Paris 1887)

Flournoy, Théodore, Des Indes à la Planète Mars. Etude sur un cas de somnambulisme avec glossolalie (Paris 2006 [1900])

Gauchet, Marcel, L'Inconscient cérébral (Paris 1992)

Ginestous, Etienne, «Les cinématophtalmies (troubles oculaires par cinéma)», Gazette hebdomadaire des Sciences médicales 23 (6 juin 1909) 265-269; 31 (1 ${ }^{\text {er }}$ août 1909) 367-368

Girard, Dr, «De l'enfance en péril moral considérée au point de vue médical», Jahrbuch der Schweizerischen Gesellschaften für Schulgesundheitspflege/Annales de la Société suisse d'hygiène scolaire (Zürich 1900) 36-72

Haugmard, Louis, «L'〈esthétique〉 du cinématographe», Le Correspondant (10 mai 1913) $762-771$

Hellwig, Albert, «Hypnotismus und Kinematograph», Zeitschrift für Psychotherapie und medizinische Psychologie 6 (1916) 310-315

Janet, Pierre, L'automatisme psychologique (Paris 1898)

Lojacono, L., «Turbe nervose consecutive alle rappresentazioni cinematografiche. Noticina clinica», Rivista italiana di neuropatologia, psichiatria ed elettroterapia (1912) 14-15

Luys, Jules, «La couronne magique. L'emmagasinement des activités cérébrales», Revue scientifique de l'occultisme. Organe mensuel de l'Evolution scientifique, littéraire et artistique, en rapport aux sciences occultes 13 (1894) 101-102

Macario, M.-M.-A., Du sommeil, des rêves et du somnambulisme dans l'état de santé et de maladie (Lyon/Paris 1857, repr. Nendeln/Liechtenstein 1978)

Masini M. U./G. Vidoni, «Il cinematografo nel campo delle malattie mentali e della criminalità», Archivio di antropologia criminale, psichiatria e medicina legale (Torino 1915) 617-629

Pailhas d'Albi, «Psychisme périodique ou cyclopsychisme», Revue de l'hypnotisme expérimental et thérapeutique 10 (1910) 302-306

Plas, Régine, Naissance d'une science humaine: la psychologie, les psychologues et le «merveilleux psychique» (Rennes 2000)

Ribot, Théodule, Les maladies de la mémoire (Paris 1897 [1881])

Richard, Nathalie, «La fin de l'homme-machine? De l'automatisme cérébral à l'inconscient psychique dans la France des années 1880», conférence tenue au Colloque international L'homme-machine et ses avatars dans la littérature des XVII au XXI siècles, Université de Lausanne/Ecole Polytechnique Fédérale de Lausanne, 22-23 mai 2008

Taine, Hippolyte, De l'intelligence, II (Paris 1883 [1870])

Tardieu, Emile, L'ennui: étude psychologique (Paris 1903)

Tollemer, Louis, «Les chocs électriques», La Presse Médicale (Samedi 7 juin 1902) 545-546

Tortajada, Maria, «Archéologie du cinéma: de l'histoire à l'épistémologie», Cinémas 14 (2004) $19-51$

Wild, A., «Bekämpfung des Kinematographen-Unwesens», Jahrbuch für Jugendfürsorge, supplément aux Annales suisses d'hygiène scolaire (Zürich 1911-1916) 